\title{
LE CONTEXTE HISTORIQUE ET ÉTHIQUE DE LA LÉGISLATION BELGE EN MATIËRE D'EUTHANASIE
}

\author{
Étienne Vermeersch
}

Fond. Nationale de Gérontologie | «Gérontologie et société »

2004/1 vol. 27 / nº 108 | pages 189 à 199

ISSN 0151-0193

Article disponible en ligne à l'adresse :

https://www.cairn.info/revue-gerontologie-et-societe1-2004-1-page-189.htm

Distribution électronique Cairn.info pour Fond. Nationale de Gérontologie.

(C) Fond. Nationale de Gérontologie. Tous droits réservés pour tous pays.

La reproduction ou représentation de cet article, notamment par photocopie, n'est autorisée que dans les limites des conditions générales d'utilisation du site ou, le cas échéant, des conditions générales de la licence souscrite par votre établissement. Toute autre reproduction ou représentation, en tout ou partie, sous quelque forme et de quelque manière que ce soit, est interdite sauf accord préalable et écrit de l'éditeur, en dehors des cas prévus par la législation en vigueur en France. Il est précisé que son stockage dans une base de données est également interdit. 


\section{- LE CONTEXTE HISTORIQUE ET ÉTHIQUE}

de la législation belge en matière d'euthanasie

ÉTIENNE VERMEERSCH

PROFESSEUR ÉMÉRITE DE L'UNIVERSITÉ DE GAND (BELGIQUE) ANCIEN PRÉSIDENT DU COMITÉ CONSULTATIF DE BIOÉTHIQUE (BELGIQUE)

L'article propose un aperçu de la genèse des lois sur l'euthanasie en Belgique et aux Pays-Bas, dans le but de donner une idée claire des concepts utilisés et des idées directrices ayant présidé à leur adoption. Le rôle du Comité Consultatif de Bioéthique dans la discussion éthique en Belgique est exposé, ainsi que le fondement de la profonde division éthique entre la "coalition gouvernementale» et les démocrates chrétiens. Après avoir récapitulé les lignes de force de chaque loi, une comparaison est faite entre la loi néerlandaise et la loi belge et les principes éthiques fondamentaux sur lesquels elles reposent.

THE BELGIAN LAW ON EUTHANASIA. THE HISTORICAL AND ETHICAL BACKGROUND

A survey is provided of the genesis of the euthanasia laws in Belgium and the Netherlands, with the intent to provide a clear idea of the concepts involved and of the rationale for their introduction.

The role of the Belgian Consultative Committee on Bioethics in the development of the ethical discussion in Belgium is explained as well as the essence of the profound ethical divide between the "coalition" and the Christian Democrats. After a summary of the basic points of the law, a comparison is made between the Belgian and the Dutch laws and their ethical foundations. 


\section{UN DÉBAT DE 30 ANS}

En Belgique, les media ont, à partir des années soixante-dix, traité occasionnellement des problèmes éthiques et légaux liés à l'euthanasie. En 1971 la télévision flamande y consacra une heure entière de débat. L'action concertée en faveur d'une législation en la matière, ne débuta toutefois que dans les années quatre-vingt, avec la fondation d'une "Association belge pour le droit de mourir dans la dignité » (1981) et de son homologue flamand, «Vereniging voor het recht op waardig sterven » (1983).

Aux Pays-Bas le débat fut ouvert dès 1971 par le docteur Geertruda Postma. Elle avait injecté une dose mortelle de morphine à sa mère, âgée de 78 ans. Celle-ci, sourde et partiellement paralysée, avait à plusieurs reprises supplié sa fille d'abréger sa vie. Après son acte, le docteur Postma en fit rapport aux autorités et elle fut poursuivi. Le jugement novateur du tribunal de Leeuwarden déclara qu'un médecin ne pouvait encourir de reproches pour avoir abrégé la vie d'un malade, à condition qu'il ait observé certains critères de prudence et dans la mesure où les conditions suivantes étaient remplies: maladie incurable, souffrances insupportables, demande expresse du patient. Une telle « abréviation de la vie " ne fut pas encore considérée comme de l'euthanasie - le tribunal condamna le docteur Postma à une peine légère - mais le débat sur le problème était définitivement ouvert.

Plusieurs autres procès ayant eu lieu, une Commission officielle fut instituée (1982) avec la mission de donner son avis en vue de l'élaboration d'une politique gouvernementale en matière d'euthanasie et de suicide assisté. Cet avis (1985) donna la définition suivante du concept d'euthanasie: "l'acte pratiqué par une personne qui met intentionnellement fin à la vie d'une autre personne qui en a fait la demande explicite». A une majorité de 13 contre 2 la commission proposa la modification de plusieurs articles du code pénal afin de rendre l'euthanasie licite, dans la mesure où elle serait exécutée par un médecin dans le cadre d'une procédure appropriée. Au mois d'octobre 1990 les procureurs généraux se mirent d'accord sur une "procédure de déclaration »: le médecin ayant pratiqué une euthanasie devait en faire la déclaration auprès du médecin légiste et lui remettre un rapport détaillé des circonstances ayant conduit à cet acte. Le médecin ne serait pas poursuivi s'il s'était conformé aux conditions prescrites. 
Une véritable législation sur l'euthanasie ne fut adoptée aux PaysBas que le 10 avril 2001 (avec entrée en vigueur le $1^{\mathrm{er}}$ avril 2002), mais l'influence exercée sur la discussion en Belgique, par l'impunité qui était ainsi accordée à une pratique bien spécifiée d'euthanasie, fut indéniable. En Belgique, la discussion politique s'amorça au milieu des années quatre-vingt-dix. Au cours de la session parlementaire de 1995-1996 des propositions de loi en matière d'euthanasie furent introduites au Sénat par quatre de ses membres. En 1996 un Comité Consultatif de Bioéthique fut fondé dans le but de conseiller les gouvernements et parlements fédéraux et communautaires à propos de problèmes bioéthiques. Ce comité, comprenant 35 membres titulaires et autant de remplaçants compte parmi ses membres: des médecins (11 parmi les titulaires, autant parmi les remplaçants), des professionnels des soins médicaux, des magistrats, des juristes, des théologiens, des moralistes et philosophes ainsi que des spécialistes en sciences sociales. Leur toute première mission, proposée par les présidents de la Chambre et du Sénat, fut de donner un " avis » au sujet des propositions de loi introduites en matière d'euthanasie. La commission rédigea deux rapports. Le premier, publié le 12 mai 1997, traitait de l'euthanasie au sens strict (sur des personnes capables), le second le 22 février 1999 se penchait sur les problèmes d'interruption de la vie de personnes incapables et des «directives anticipées » établies par des personnes capables préalablement à une éventuelle incapacité (l'expression " testament de vie " pour ces directives fut rejetée comme étant «équivoque »).

Les membres du Comité durent constater leur désaccord interne en ce qui concerne les questions fondamentales, mais il est important de noter qu'il y eut une complète unanimité sur les points suivants :

a) La définition néerlandaise fut adoptée: «l'euthanasie est l'acte pratiqué par un tiers qui met intentionnellement fin à la vie d'une personne à la demande de celle-ci ».

b) Par conséquent cette définition ne s'appliquait pas aux personnes incapables, pour lesquelles la terminologie suivante fut adoptée: "L'arrêt actif de la vie d'une personne incapable est l'acte pratiqué par un tiers qui met intentionnellement fin à la vie d'une personne incapable d'exprimer sa volonté. ».

c) Plus important encore, le fait d'arrêter un traitement devenu vain («futile ») n'était pas considéré comme étant un acte d'eutha- 
nasie et la recommandation fut faite de ne plus utiliser pour ces cas l'expression "euthanasie passive »;

d) Ce qui est parfois à tort appelé « euthanasie indirecte ", i.e. l'augmentation des doses de calmants ou d'analgésiques pouvant avoir comme effet secondaire d'abréger la vie, était également clairement distingué de l'euthanasie.

Il est regrettable qu'un certain nombre de médecins, même parmi ceux qui sont régulièrement confrontés aux situations décrites sous (c) et (d), semblent encore penser que la loi sur l'euthanasie serait applicable aux décisions à prendre dans de tels cas. Il y avait unanimité parmi les membres du Comité que des «décisions en fin de vie » de cette nature ne peuvent être considérées comme "l'acte de mettre fin à la vie de quelqu'un " (et ne devraient donc pas jeter le trouble dans les discussions concernant l'euthanasie proprement dite). Certains proposèrent l'élaboration de procédures légales pour la prise des décisions dans de tels cas, tandis que d'autres étaient d'avis que l'on pouvait faire confiance aux spécialistes afin d'instituer des règles, en accord avec les comités éthiques de leurs instituts médicaux (par exemple, les directives concernant les réanimations).

Sur l'essentiel de la question, trois opinions fondamentales furent formulées: certains membres se déclarèrent adversaires inconditionnels de l'euthanasie, qu'ils estimaient en contradiction avec la valeur inaliénable de la vie d'une personne humaine; d'autres pensaient que l'euthanasie devait être admise, par compassion et par respect pour l'autodétermination de la personne. Un groupe de membres - certains d'entre eux d'inspiration chrétienne - se situant à égale distance de ces deux thèses, proposa que l'euthanasie soit autorisée dans des cas de force majeure, lorsque deux devoirs du médecin s'opposent (le devoir de préserver la vie et celui de soulager des souffrances insoutenables). Ces trois opinions fondamentales aboutirent aux quatre propositions différentes:

a) Une modification législative dépénalisant l'euthanasie.

b) Une régularisation procédurale a posteriori d'une euthanasie décidée en colloque singulier entre le médecin et le patient. Cette approche s'aligna sur le modèle du compromis hollandais en vigueur à ce moment-là, qui proposait un maintien symbolique de l'interdit pénal de l'euthanasie, tout en définissant un certain nombre de "critères de prudence" que le médecin devrait res- 
pecter afin d'éviter une poursuite par les procureurs généraux. Les mêmes critères étaient d'ailleurs proposés par ceux qui étaient favorables à un changement de la loi. Dans les deux cas le médecin aurait à demander l'avis d'un collègue et devrait, après l'acte, rédiger un rapport et le transmettre aux autorités légales. Le fait que le contrôle serait réalisé après l'acte, fut à l'origine de l'expression "procédure a posteriori ».

c) Lors d'une "procédure a priori ", toute décision concernant un acte d'euthanasie devrait être précédée d'un débat éthique entre le médecin, un second médecin, la famille, l'équipe d'assistants médicaux et un expert en sciences éthiques; dans les cas où l'euthanasie serait décidée, le médecin en avertirait les autorités judiciaires et plaiderait le cas de force majeure. Évidemment dans chacun des cas proposés, l'euthanasie ne pourrait être réalisée que dans la mesure où le médecin aurait acquis la certitude que le malade, dûment informé, était dans un état de souffrance insoutenable et que sa demande d'abréger sa vie était faite de son plein gré.

d) Le maintien pur et simple de l'interdit légal contre l'euthanasie.

Sur la base de cet avis, le Sénat tint un débat les 9 et 10 décembre 1997. Aucun résultat ne fut atteint avant les élections de 1999. Le problème majeur était que les démocrates chrétiens ne pouvaient accepter de légiférer que dans la mesure où rien ne serait modifié au code pénal, acceptant seulement la possibilité de faire appel à la "force majeure" dans les cas extrêmes, tandis que les autres partis voulaient aller plus loin. Le parti d'extrême-droite, Vlaams Blok demeura isolé dans son refus de tout changement.

A l'issue des élections de 1999, un gouvernement de coalition fut formé par les libéraux, les socialistes et les verts, reléguant les démocrates chrétiens dans l'opposition. Entre-temps (février 1999) $l^{\prime} A v i s\left(n^{\circ} 9\right)$ du Comité Consultatif de Bioéthique, "concernant l'arrêt actif de la vie des personnes incapables d'exprimer leur volonté » était paru. Il avait abouti à trois propositions divergentes:

a) Une reconnaissance légale de l'arrêt actif de la vie des incapables dans un certain nombre de cas, à condition que la personne concernée ait rédigé une "déclaration anticipée" allant dans ce sens.

b) La régulation procédurale a priori de l'arrêt actif de la vie, demandé par le patient dans une directive anticipée. 
c) Le maintien pur et simple de l'interdit légal de tout arrêt actif de la vie.

Peu de temps après, plusieurs sénateurs, représentant chacun un des partis de la coalition, introduisirent une proposition de loi inspirée en partie par la proposition (a) contenue dans les deux Avis émis par le Comité Consultatif de Bioéthique. Les démocrates chrétiens introduisirent de leur côté une proposition basée sur la proposition (c) du premier de ces Avis. Certains responsables politiques craignaient que la loi belge sur l'euthanasie puisse bien constituer une première mondiale et pour cette raison espéraient parvenir à un compromis qui permettrait d'adopter la loi à une grande majorité. Toutefois, après une période de réflexion de deux mois (avril - mai 2000) et suite à une quarantaine de séances d'audition d'experts, le seul résultat fut un nombre accru de propositions d'amendement au texte de la proposition de loi de la majorité. Le "grand compromis » ne fut dès lors pas réalisé.

La proposition (c) du premier avis du Comité Consultatif, contenant les idées de base de procédure a priori et de force majeure avait laissé entrevoir la possibilité d'un accord avec les démocrates chrétiens, mais il apparut bientôt qu'une partie des membres de ce parti demeurait opposée à toute forme d'euthanasie et en tout cas à une modification de la loi. Certains démocrates chrétiens progressistes étaient, quant à eux, conscients du fait qu'une assez grande majorité se dessinait au sein de la population en faveur de l'euthanasie. En effet, beaucoup de personnes ont été confrontées avec la souffrance insoutenable de membres de leur famille ou d'amis. Néanmoins ces chrétiens préférèrent privilégier ce qu'ils considèrent comme une des valeurs fondamentales de la tradition chrétienne: le respect absolu de la vie humaine. Dans l'Exposé des motifs de la proposition de loi introduite par le CVP (chrétiens démocrates flamands) nous lisons: "Cette tradition séculaire du respect de la vie est devenue une norme dans notre civilisation occidentale, même pour ceux qui rejettent la croyance en Dieu. Chaque vie est unique et mérite estime, respect, protection. La nécessité de protéger la vie se ressent le plus fortement au cours du processus menant à la mort. La valeur de la vie ne dépend pas d'un jugement individuel ».

Une telle approche se caractérise par un choix de principe pour l'hétéronomie, - en opposition avec ceux qui jugent l'autonomie de la personne comme étant la valeur centrale - i.e. que l'origine 
de la valeur de la vie et de la personne humaine ne se retrouve pas dans cette personne elle-même (autos), mais auprès d'une autorité différente (hétéros). Étant donné que la thèse selon laquelle Dieu est cette autre autorité, n'est plus universellement acceptée, il y a une tendance à la remplacer par la société : «Tout comme la naissance, le décès est un événement social. Lorsque la personne humaine se rapproche de la mort sans qu'une main amicale ne lui vienne en aide, le caractère social du passage de la vie à la mort est nié." (ibidem).

En partant d'un tel point de vue, l'on ne pourra jamais accepter qu'un texte de loi, qui est d'application générale, et plus particulièrement les articles du Code pénal qui traitent du fait de tuer, puissent autoriser de mettre fin à la vie de quelqu'un. Cela voudrait dire que l'autonomie, le droit à l'autodétermination, deviendrait d'une certaine manière une valeur en soi et qu'elle éliminerait la prééminence de l'autorité hétéronome. «Le devoir qu'ont les autorités de protéger la vie humaine est fondé sur la conviction que la vie humaine représente une valeur, rien que du seul point de vue de la dignité humaine, ce qui veut dire qu'elle est un bienfait en soi, quel que soit l'accord ou le désaccord de l'individu. » (ibidem).

Même lorsque la vie est devenue sans objet et insupportable pour la personne concernée, elle s'impose à lui comme un fait inéluctable, revêtu de l'auréole de la "dignité humaine». Ce point de vue, qui, en ce qui concerne ses prémisses, équivaut à celui tenu par les opposants inconditionnels à toute euthanasie, a néanmoins, aussi bien dans la proposition (c) citée plus haut que dans la proposition de loi des démocrates chrétiens, obtenu une expression plus humaine, puisqu'on y accepte qu'en des circonstances exceptionnelles le médecin puisse être confronté à une situation de détresse, engendrant un conflit entre deux valeurs, ce qui l'oblige à faire un choix. A noter toutefois que dans un tel contexte, le droit du malade à l'autodétermination n'est pas pris en compte: le problème de l'euthanasie est considéré essentiellement comme un dilemme moral qui se pose pour le médecin. De la sorte, le principe de base de l'hétéronomie est sauf.

Le désaccord fondamental porte sur l'essence même de la condition humaine: le rôle central de l'autorité hétéronome d'une part et l'accent mis sur l'autonomie et sur le respect et la réserve en face de la souffrance humaine d'autre part. Ce différend explique pour- 
quoi en fin de compte tout compromis fut impossible, à moins que l'un des deux groupes antagonistes n'accepte de renier ses principes fondamentaux. En effet, au-delà des nuances d'opinion qui pouvaient exister parmi les membres de la majorité, tous se ralliaient à l'idée que l'acceptation de l'euthanasie était fondée:

a) Sur le droit fondamental de toute personne humaine à l'autonomie: chaque personne a droit à l'autodétermination concernant sa propre santé et sa vie, en particulier lorsqu'elle se trouve confrontée à des souffrances insoutenables.

b) Sur le fait que dans la mesure où le médecin, en pratiquant l'euthanasie, exprime sa compassion et sa solidarité avec la personne souffrante, son acte devrait être considéré comme une intervention d'une haute qualité éthique.

c) Sur la considération que les personnes adhérant à une autre vision de la vie humaine devraient, dans une société pluraliste, respecter les valeurs des autres et leur permettre d'agir en accord avec ces valeurs, pour autant que, ce faisant, d'autres personnes ne soient pas lésées.

\section{LA LOI DU 16 MAI 2002}

Une fois ces valeurs admises en tant que principes fondamentaux, il ne restait plus au législateur qu'à mettre au point des règles prenant en compte les droits acceptés, tout en empêchant toute forme d'abus.

La loi tendant à satisfaire à ces conditions fut votée au Sénat par les commissions conjointes de la Justice et des Affaires sociales, le 20 mars 2001 (après avoir délibéré au sujet de 687 amendements); ensuite en séance plénière du Sénat le 25 octobre 2001 et enfin à la Chambre le 16 mai 2002 (par 86 voix contre 51 et 10 abstentions). La loi est entrée en vigueur le 23 septembre 2002.

Les points essentiels de cette loi sont qu'un médecin (et personne d'autre) peut pratiquer un acte d'euthanasie sans pour autant commettre de crime, lorsque :

- le malade qui le lui demande a l'âge requis, est légalement compétent et est conscient ;

- la demande est spontanée, bien réfléchie et exprimée à plusieurs reprises; 
- le malade endure des souffrances physiques ou psychologiques insoutenables et prolongées, à la suite d'une situation pathologique incurable, causée par une maladie ou un accident.

Le malade doit être informé sur son état de santé et son espérance de vie, tandis que le médecin doit avoir acquis la conviction que la requête du malade est sincère. Un deuxième médecin sera consulté dans le but de vérifier ces différents éléments. Dans la mesure du possible, le problème sera discuté avec le personnel assurant l'assistance médicale et avec les proches du malade. Lorsque le malade n'est pas dans une phase "terminale ", i.e. qu'il n'est pas prévu qu'il ou elle meure très prochainement de mort naturelle, un troisième médecin sera consulté et une période d'au moins un mois sera respectée entre la requête par écrit du malade et l'acte d'euthanasie. Mettre fin à la vie d'une personne inconsciente peut également se faire, lorsqu'elle a établi une "déclaration anticipée » concernant l'euthanasie. Ce document doit être établi au cours des cinq années précédentes. Après avoir pratiqué l'euthanasie, le médecin devra en faire rapport auprès de la Commission fédérale de contrôle et d'évaluation.

Étant donné que les discussions des points de vue éthiques ayant précédé l'adoption des lois - y compris la publication de données et de chiffres pertinents - ont été pratiquement identiques en Belgique et aux Pays-Bas, il nous semble instructif de comparer les deux lois. A mon avis la loi néerlandaise est de loin la meilleure, non seulement parce qu'elle est la plus "libérale» - ce qui est indéniable, même si cela est parfois nié lors de certaines discussions politiques - mais surtout parce qu'elle est plus cohérente, tant du point de vue moral que légal.

Le premier élément apparaît clairement à propos du traitement divergent réservé aux enfants et en général aux mineurs d'âge. La loi néerlandaise accepte la requête d'euthanasie faite de façon indépendante dès l'âge de 16 ans, et moyennant le consentement des parents, entre l'âge de 12 à 16 ans. Ainsi est pris en compte le fait que les souffrances insoutenables endurées par des mineurs peuvent être tout aussi horribles que celles des adultes et que la capacité à l'autodétermination est une qualité variable qui gagne en importance dans la mesure où l'on est davantage confronté à ces souffrances. En Belgique rien n'est possible pour les mineurs d'âge, malgré le fait que jamais la moindre argumentation morale sensée n'ait été avancée en faveur de cette omission. 
La supériorité de la loi néerlandaise se manifeste d'un point de vue juridique par le fait qu'aux Pays-Bas le concept (non point le terme lui-même) d'euthanasie est clairement introduit dans le Code pénal. Il s'agit là évidemment d'un souci louable d'honnêteté: si la société décide qu'une exception importante peut être acceptée en ce qui concerne l'interdiction générale de tuer, (en-dehors des cas de légitime défense), elle doit l'ajouter explicitement en tant que restriction à la loi universelle. Ce qui permet en même temps d'indiquer les peines spécifiques encourues lors d'une application désinvolte de la loi. En Belgique, l'euthanasie a été introduite grâce à une loi séparée, dans le but (pas très honnête à mon avis) de donner l'impression que l'interdiction générale est toujours maintenue, et avec l'effet secondaire que chaque manquement par rapport aux critères de prudence sera poursuivie comme un meurtre.

Une autre différence notable entre les deux lois, provenant d'appréciations divergentes dans l'opinion publique des deux pays, concerne les malades "non moribonds ». Aux Pays-Bas, les thèmes fondamentaux dans la discussion sur l'euthanasie furent toujours l'autonomie du malade et la compassion avec ses souffrances: que la mort naturelle fût ou non proche, n'était pas vraiment considéré comme un élément important; aussi la loi n'a-t-elle pas introduit cette distinction. En Belgique l'opposition la plus virulente à la proposition de loi de la coalition se concentra sur le fait que l'euthanasie pourrait également $s$ 'appliquer à des malades «non mourants ». La raison semble à nouveau devoir être recherchée dans l'attitude (chrétienne) frileuse à l'encontre de l'autodétermination. Dans le cas d'une maladie "terminale ", la mort elle-même est causée par un agent " hétéronome ", i.e. la nature, le seul effet de l'euthanasie étant d'accélérer le processus. Lorsqu'il s'agit d'une maladie "non terminale", la décision est entièrement entre les mains du malade. L'opposition à cet aspect fut tellement forte que le législateur se vit forcé de prévoir en de tels cas l'avis d'un troisième médecin et un délai d'attente d'un mois.

Un dernier point qui fut l'objet de grands débats en Belgique, concernait la clause prévoyant que le médecin pourrait prendre en compte « des souffrances insupportables et durables, physiques ou psychologiques" (souligné par moi). L'objection, venant surtout des milieux médicaux, était qu'ainsi la possibilité était offerte de pratiquer l'euthanasie dans des cas d'extrême détresse ou de 
dépression, en l'absence de troubles somatiques. Il s'agissait là toutefois d'une interprétation erronée, puisque la loi prévoit explicitement comme condition une situation pathologique incurable, causée par la maladie ou un accident. En outre, lorsque le malade ne se trouve pas dans une phase terminale, l'avis d'un troisième médecin (dans les cas de dépression, un psychiatre) doit être sollicité. Il est évident que dans la plupart des cas de ce type, ce médecin conclura, ou bien qu'il ne s'agit pas d'un patient vraiment capable (par exemple, lorsqu'il s'agit d'une grave dépression pathologique) ou qu'il n'a pas constaté une maladie incurable et qu'il n'y a donc pas lieu de pratiquer l'euthanasie.

Pourquoi dès lors prendre également en charge la "souffrance psychologique "? Parce que, dès qu'une maladie incurable a été diagnostiquée, la question de savoir si le malade ressent la situation comme insupportable et sans espoir dépend également d'aspects psychologiques. La perspective de traîner une vie diminuée et humiliante peut pour un certain nombre de personnes constituer une raison suffisante pour demander la grâce de la mort.

La loi néerlandaise ne mentionne pas les "souffrances psychologiques ", mais les décisions des tribunaux et les rapports des comités d'évaluation démontrent clairement que ces aspects du problème tel qu'il est ressenti par les malades, sont dûment pris en compte.

Il semble évident qu'aux Pays-Bas, où une discussion approfondie au sujet de l'euthanasie s'instaura plus de dix ans avant, une loi plus cohérente et mieux équilibrée a pu être réalisée. Nous formons néanmoins l'espoir que la loi belge, en dépit de ses lacunes, sera désormais en mesure d'offrir la solution de dernier ressort à la plupart des personnes qui préfèrent une mort délibérément choisie plutôt que des souffrances insoutenables. 